\title{
Efficacy of Bifidobacterium animalis subsp. lactis BB-12 against Giardia duodenalis trophozoites: an experimental study
}

\author{
Fahu Yuan ${ }^{1}$, Yufei Liu ${ }^{1}$, Qian Gui ${ }^{1}$, Qiuyi Huang ${ }^{1}$, Qianyu Li ${ }^{1}$, Xuping Yang ${ }^{1}$, Lixin Qiu ${ }^{1}$, Jinmei Feng ${ }^{1, *}, X_{i j i}$ Shu $^{2, *}$ \\ ${ }^{1}$ Department of Pathogenic Biology, School of medicine, Jianghan University, Wuhan 430056, China \\ ${ }^{2}$ Department of Pathology and Pathophysiology, School of medicine, Jianghan University, Wuhan 430056, China
}

\begin{abstract}
Giardia duodenalis, formerly known as Giardia lamblia, is an important zoonotic protozoan parasite. It mainly infects the intestines of humans, dogs, cats and domestic animals, causing diarrhea, abdominal pain, indigestion and weight loss. At present, all the clinical drugs for the treatment of Giardia have problems such as side effects and drug resistance to varying degrees, and the development of new drugs for the treatment of Giardia is still a hot issue. There is growing interest in using probiotics as an antiintestinal parasite strategy. The present study aimed to assess the effect of supernatants of Bifidobacterium Animalis Subsp. lactis $B B-12$ on giardia the growth of giardia trophozoites. In this study, the Bifidobacterium animalis subsp. lactis BB-12 were cultured in BBL liquid medium, and the effects of the supernatants on the growth and adhesion of trophozoites of Giardia were observed. The results showed that the growth of Giardia flagellate was significantly inhibited by the supernatant. The influence of the supernatant on the morphology of the trophozoites was observed by microscope, and it was found that the surface of the trophozoites was uneven, the shape was atrophied, the surface cell membrane was broken to some extent, and the contents were spilt. In summary, the results of this study suggest that the fresh-cultured supernatants of the probiotic Bifidobacterium Animalis subsp. lactis BB-12 have anti-Giardia effects.
\end{abstract}

\section{Introduction}

Giardia duodenalis is a parasite of the upper segment of the small intestine of humans and various mammals, which can cause giardiasis with main symptoms such as abdominal pain, diarrhea, flatulence, indigestion and weight loss. It has become an important opportunistic pathogenic pathogen in the world and is also one of the ten major parasitic diseases that seriously threaten human public health [1].

Giardiasis is a zoonotic parasitic gastrointestinal disease caused by Giardia parasiting in the small intestine of human, dog, cat and many mammals. Giardia is a common parasite that causes diarrhea and gastrointestinal diseases in developed countries, especially in the United States, where it was once called the "number one intestinal parasite". Giardiasis in China is also quite popular, especially dog giardia infection more, greater harm to puppies. Dogs are in close contact with human beings, which can easily cause the epidemic of giardiasis, which poses a serious threat to human health and public health safety. The infection rate is around $20 \%$ in all regions of the country. Studies have reported that Giardia is more prevalent in AIDS patients, and has become one of the opportunistic infection protozoa, and patients with severe persistent diarrhea after infection. In recent years, giardiasis has attracted increasing attention in the field of biology [2].
At present, the effective drugs for the treatment of giardiasis are metronidazole, tinidazole and albendazole. The preferred anti-Giardia drug is metronidazole, whose mechanism of action is to inhibit the protein synthesis and REDOX reaction or nitrogen chain fracture of the trophozoite, so that the worm body cannot be metabolized normally, resulting in the stop of growth and reproduction and deformity, and finally death. In addition, since giardia is an opportunistic pathogenic parasite, the organism may be recessive in infection. In vivo studies have shown that metronidazole can not eliminate $100 \%$ of Giardia, the body infection with Giardia only does not show obvious clinical symptoms. Although metronidazole has a good curative effect, its side effects are significant. It is easy to develop drug resistance, greater damage to the digestive system, and there are obvious adverse reactions such as gastrointestinal bleeding. The drug also has teratogenic and mutagenic effects, which largely restrict use of metronidazole in the clinical. Therefore, the development of new drugs against giardiasis is still a hot issue [3].

Some recent in vitro and in vivo data suggest that probiotic treatment may reduce giardia infection and improve symptoms of giardiasis. Probiotics, due to their high activity, stability and low toxicity to human and other mammalian hosts, have attracted much attention as potential alternatives or combination therapies against Giardia [4].

\footnotetext{
* Corresponding author: fengjm@jhun.edu.cn; shuxiji@sina.com
} 
In the present study, potential negative effects of the supernatant of Bifidobacterium animalis subsp. lactis $B B-12$ on Giardia duodenalis were evaluated in vitro.

\section{Material and method}

\subsection{Bifidobacterium Animalis subsp. lactis BB- 12}

Bifidobacterium Animalis subsp. lactis BB-12 (BB-12) was obtained from Chr. Hansen (China) Co., LTD., and was cultured in improved MRS medium. The medium was formulated with tryptone $20 \mathrm{~g}$, glucose $20 \mathrm{~g}$, triammonium citrate $2 \mathrm{~g}$, sodium acetate $5 \mathrm{~g}$, Tween- 80 $1 \mathrm{~mL}, \mathrm{~K}_{2} \mathrm{HPO}_{4} 2 \mathrm{~g}, \mathrm{MgSO}_{4} \cdot 7 \mathrm{H}_{2} \mathrm{O} 0.2 \mathrm{~g}, \mathrm{MnSO}_{4} \cdot \mathrm{H}_{2} \mathrm{O}$ $0.05 \mathrm{~g}$, L-cysteine hydrochloride $1.0 \mathrm{~g}$, distilled water added to the volume to $1 \mathrm{~L}$, acetic acid adjusted $\mathrm{pH}$ value of 7 .

\subsection{Pure culture of Giardia trophozoites}

Take out the frozen storage tube containing Giardia lamblia from the liquid nitrogen tank, put it in the constant temperature water bath pot at $37{ }^{\circ} \mathrm{C}$, and quickly melt it. Then transfer it to the culture tube in the ultra-clean table, and finally fill the culture bottle with the improved TYI-S-33 medium, and place it in the constant temperature incubator at $37{ }^{\circ} \mathrm{C}$. When Giardia trophozoites is cultured to the logarithmic growth stage, that is, the culture tube is full of Giardia, and the passage of giardia can be carried out.

The culture tube full of Giardia trophozoites was placed on ice for ice bath. After 15 minutes, the tube was taken out and rolled by hand for several minutes, so that all the Giardia trophozoites attached to the tube fell off into the culture medium of Giardia. The trophozoites and the medium were transferred to the centrifuge tube for centrifuging at $3500 \mathrm{r} / \mathrm{min}$ for 10 minutes. The supernatant was discarded, and then the fresh medium was added to resuspend the precipitation. Finally, the concentration of trophozoites was adjusted to $1 \times 10^{6}$ trophozoites $/ \mathrm{mL}$ with the blood cell count plate, and used as inoculum to study the in vitro effects of BB-12 supernatants on growth and adherence of Giardia duodenalis trophozoites.

\subsection{Effects of BB-12 supernatant in vitro}

The growth and adhesion of BB-12 supernatant to Giardia duodenalis trophozoites in vitro was evaluated using previously reported method $\mathrm{s}$ [4]. In brief, the supernatant was obtained by growing BB-12 (10 $10^{1}$ UFC) in antibiotic-free TYI-S-33 medium for $24 \mathrm{~h}$ at $37{ }^{\circ} \mathrm{C}$. The supernatant obtained from these cultures (BB-12) was collected and centrifuged at $4,000 \mathrm{~g} \times 10 \mathrm{~min}$ using a $0.22 \mu \mathrm{m}$ filter and $\mathrm{pH}$ adjusted to 7 using $5 \mathrm{~N} \mathrm{NaOH}$. The supernatant was used as fresh (BB-12 fresh) and after 30 minutes of heat treatment at $56^{\circ} \mathrm{C}\left(\mathrm{BB}-1256^{\circ} \mathrm{C}\right)$ and $90^{\circ} \mathrm{C}$ (BB-12 $\left.95{ }^{\circ} \mathrm{C}\right)$. For all tests, $100 \mu \mathrm{L}$ BB-12 supernatant was added to $900 \mu \mathrm{L}$ fresh TYI-S-33 medium in a $1.5 \mathrm{~mL}$ eppendorf vial with $5 \times 10^{4}$ logarithmic trophozoite (BB-12 FS treatment group). The negative control (NC) was carried out under similar experimental conditions without any supernatant.

\subsection{Growth inhibition assay}

The growth of Giardia duodenalis trophozoites was evaluated in fresh (BB-12 fresh) and heat-treated (BB-12 $56{ }^{\circ} \mathrm{C}$ and BB-12 $95^{\circ} \mathrm{C}$ ) BB-12 supernatant cultures and in negative control for $24 \mathrm{~h}$ and $48 \mathrm{~h}$, respectively. After each different incubation period, the culture flask was placed at $4{ }^{\circ} \mathrm{C}$ for 15 minutes, the trophozoites were suspended, and the total number of cells was counted in triplicates under an inverted microscope using the blood cell counting board counting chamber.

\subsection{Adhesion inhibition measurement}

To assess the effect of BB-12 supernatant (BB-12 $56{ }^{\circ} \mathrm{C}$ and BB-12 $95{ }^{\circ} \mathrm{C}$ ) on Giardia duodenalis trophozoites adhesion capacity, trophozoites were evaluated 24 and 48 hours later and compared with those observed in negative controls. After inverted mixing, $10 \mu \mathrm{L}$ culture medium was taken from each culture and the number of unattached cells was counted in triplicate using a blood cell counting plate under an optical microscope (Nikon T100). After 15 minutes of exposure at $4{ }^{\circ} \mathrm{C}$, the total number of cells was calculated using the growth assay. The result is expressed as the percentage of the attached trophozoites relative to the total Giardia duodenalis trophozoites. More specifically, these percentages were obtained by dividing the difference between the number of trophozoites counted in the medium after 15 minutes of exposure to the medium at $4{ }^{\circ} \mathrm{C}$ (total cells) and the number of trophozoites counted in the medium at $37{ }^{\circ} \mathrm{C}$ (non-adherent cells).

\subsection{Giemsa staining and morphological observation of the giardia trophozoites}

The Giardia duodenalis trophozoites were cultured in modified TYI-S-33 medium. The trophozoites were collected, centrifuged at $3500 \mathrm{r} / \mathrm{min}$ for $10 \mathrm{~min}$, rinsed and precipitated, and made into suspension drops on the slide. After natural drying, Giemsa staining solution was used for staining for $15 \mathrm{~min}$, the staining solution was discarded, and then rinsed with tap water. After drying, the slides were sealed for observation under an optical microscope.

\subsection{Statistical analysis}

The experimental data is expressed as the mean \pm standard deviation, and SPSS 13.0 is used to perform a one-way analysis of variance on the experimental data. $P<0.05$ is considered as a significant difference. 


\section{Results}

\subsection{BB-12 supernatant inhibited the proliferation of giardia trophozoites in vitro}

In the growth inhibition test, BB-12 fresh supernatant inhibited the growth of giardia compared with the untreated control group. After 48 hours of treatment with fresh BB-12 supernatant, it was found that the number of trophozoites in the BB-12 Fresh group $\left(22.35 \pm 9.07 \times 10^{4}\right)$ was significantly lower than that in the normal control group $\left(58.25 \pm 9.58 \times 10^{4}\right)(P<0.05)$ (Fig.1).
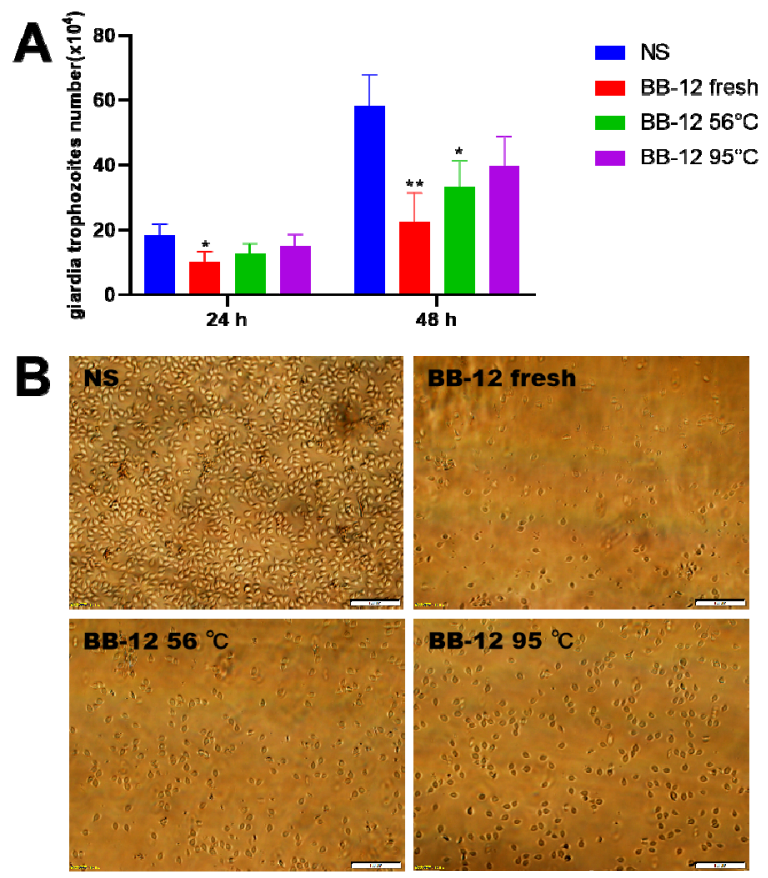

Fig. 1. Bifidobacterium Animalis subsp. lactis BB-12 supernatant inhibited the proliferation of Giardia trophozoites in vitro.

A. Growth inhibition of Giardia duodenalis trophozoites by fresh (BB- 12 fresh) and $56^{\circ} \mathrm{C}\left(\mathrm{BB}-1256^{\circ} \mathrm{C}\right)$ and $95^{\circ} \mathrm{C}(\mathrm{BB}-$ $\left.1295^{\circ} \mathrm{C}\right)$ heat-treated BB-12 supernatants. The number $\left(\times 10^{4}\right)$ of Giardia duodenalis trophozoites are expressed as average and standard deviation of trophozoites counted in three replicates after 24 and $48 \mathrm{~h}$ observation periods. $* P<0.05$, $* * P<0.01$ compared with NS group.

B. The growth appearance of each group under optical microscope for a $48 \mathrm{~h}$-period treatment.

\subsection{BB-12 supernatant inhibited the adhesion ability of Giardia trophozoites in vitro}

In the adhesion test, after a $24 \mathrm{~h}$-period of treatment, the trophozoites observed adhering to the wall of the culture tube in the BB-12 fresh treatment group (13.22 $5.34 \%)$ were significantly lower than that observed in the untreated culture $(56.12 \pm 8.07 \%)(P<0.01)$. Moreover, after 48 hours of treatment, the number of trophozoites in the BB-12 fresh treated culture $(10.85 \pm 3.66 \%)$ was still significantly lower than that in the untreated culture

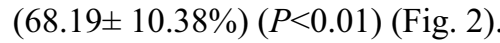

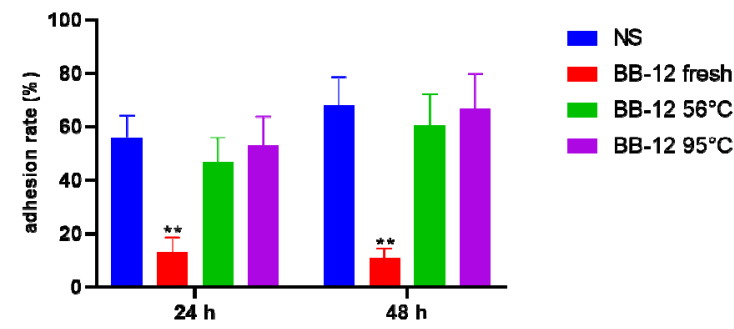

Fig. 2. Adhesion inhibition of Giardia duodenalis trophozoites by fresh (BB- 12 fresh) and $56^{\circ} \mathrm{C}\left(\mathrm{BB}-1256^{\circ} \mathrm{C}\right)$ and $95^{\circ} \mathrm{C}$ (BB$1295^{\circ} \mathrm{C}$ ) heat-treated BB-12 supernatants.

$* * P<0.01$ compared with NS group.

\subsection{Effects of BB-12 on morphology of Giardia trophozoites}

As shown in Figure 3, the trophozoites of Giardia in the control group were inverted in the shape of half-cut pears. The trophozoites were symmetrical on both sides, with a rounded front and a pointed rear. And the abdomen is flat, a slight bulge at the back, there are 4 pairs of flagella, the nuclear membrane of the trophozoite is intact and the boundary is clear. After BB12 fresh supernatants treated $48 \mathrm{~h}$, its structure deformation condition, nourishes the body stop dividing, circle, the surface is uneven, and flagella have a certain degree of damage or loss, the part of the pathological changes significantly in the trophozoite, nourishes the body near the ventral sucker and absorbing of surface is the main pathological changes caused by the membrane on the surface of the ventral sucker rupture, lead to nourish the cell contents leaked out, and other parts of the surface film shrinking situation, the changes are due to trophozoite nourishes the body the three-dimensional structure of a big change, some seriously damaged and nourish the body surface due to the cytoplasmic contents spill over and cytoplasmic draining and lose their support, flattening and cracking, resulting in death (Fig. $3)$.

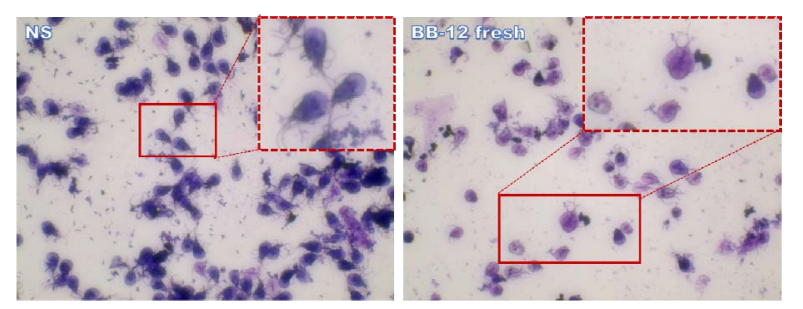

Fig. 3. Images of the Giardia trophozoites after a $48 \mathrm{~h}$ treatment of BB-12 fresh supernatants, stained by Giemsa stain.

\section{Discussion}

Giardia is a common fecal-oral parasite and one of the most important causes of diarrhoeal disease in humans and animals worldwide. Indeed, giardia infection can be asymptomatic, or cause diarrhea, dehydration, intestinal malabsorption, and chronic fatigue. Postinfective irritable bowel syndrome and intestinal malnutrition have also been shown to be possible consequences of giardiasis in humans. Most of the drugs used for the 
treatment of giardiasis have various defects, which makes the development of new drugs to inhibit the killing of Giardia become the focus of current research.

Probiotics produce compounds that inhibit pathogens such as viruses, bacteria, fungi, parasites and cancer cells. In fact, bacterins derived from Lactobacillus acidophilus have been found to inhibit the adhesion and growth of Giardia in vitro. Decreased intestinal parasite density and intestinal pathologic changes in mice infected with Giardia after Lactobacillus acidophilus bacteriocins treated [5].

Consistent with previous studies [6], some probiotic metabolites can induce morphological changes in the trophozoites of Giardia. The inhibition of Giardia shown in the fresh supernatant of BB-12 was greatly reduced by heat treatment at $56{ }^{\circ} \mathrm{C}$ and completely disappeared at $95{ }^{\circ} \mathrm{C}$, indicating that the active metabolites contained in the supernatant of BB-12 were likely to be heat-unstable compounds.

\section{Conclusion}

In conclusion, the results of this study suggest that fresh supernatant of commercial probiotics BB-12 has a negative effect on Giardia trophozoites and can be used to prevent and/or reduce the pathogenic effects of Giardia and the proliferation of this protozoan parasite in infected hosts. However, further research is needed to assess its efficacy in infected animals.

\section{Acknowledgments}

The authors are indebted to Prof. Xianmin Feng (Jilin Medical College) for kindly providing the Giardia lamblia. This work was supported by the Natural Science Foundation of China (Project No. 32070420). This project was funded by Grants from the Guiding Project in Science Research Program from Education Department of Hubei (B2020222).

\section{References}

1. Amer E I, Mossallam S F, Mahrous H. Therapeutic enhancement of newly derived bacteriocins against Giardia lamblia[J]. Experimental Parasitology, 2014, 146:52-63.

2. Pintong A R, Ruangsittichai J, Ampawong S, et al. Efficacy of Ageratum conyzoides extracts against Giardia duodenalis trophozoites: an experimental study[J]. BMC Complementary Medicine and Therapies, 2020, 20(1).

3. Vivancos V, González-Alvarez, I, Bermejo M, et al. Giardiasis: Characteristics, Pathogenesis and New Insights About Treatment[J]. Current Topics in Medicinal Chemistry, 2018.

4. Leung A, Leung A, Wong A, et al. Giardiasis: An Overview[J]. Recent Patents on Inflammation \& Allergy Drug Discovery, 2019, 13(2):134-143.
5. Ganguly S, Raj D, Gupta V K, et al. Giardia and giardiasis: an overview of recent developments.[J]. 2016.

6. Perrucci Stefania, Fichi Gianluca, Ricci Enrica et al. In vitro and ex vivo evaluation of the anti-Giardia duodenalis activity of the supernatant of Slab51 (SivoMixx).[J] .PLoS One, 2019, 14: e0213385. 